\title{
Post-application of dry vortex grinding improves the yield of a $[2+2]$ photodimerization: addressing static disorder in a cocrystal
}

\author{
Elizabeth Elacqua, ${ }^{1}$ Katherine A. Kummer, ${ }^{2}$ Ryan H. Groeneman, ${ }^{2 *}$ Eric W. \\ Reinheimer, ${ }^{3}$ and Leonard R. MacGillivray ${ }^{1 *}$ \\ ${ }^{1}$ Department of Chemistry, University of Iowa, Iowa City, IA 52242 USA; Email: len- \\ macgillivray@uiowa.edu
}

${ }^{2}$ Department of Biological Sciences, Webster University, St. Louis, MO, 63119, USA; Email: ryangroeneman19@webster.edu

${ }^{3}$ Department of Chemistry and the W.M. Keck Foundation Center for Molecular Structure, California State University, San Marcos, CA, 92096, USA

\begin{abstract}
A post-application of dry vortex grinding is used to improve a solid-state photodimerization to near quantitative yield that without grinding does not go to completion. Variable temperature Xray crystal structure data support the vortex grinding to overcome detrimental effects of static disorder that suppresses the yield of the photoreaction in the solid.
\end{abstract}

\section{Introduction}

We have demonstrated that mechanochemistry in the form of dry vortex grinding provides a means to form crystalline organic materials for applications in chemical reactivity and catalysis [1]. The mechanochemical approach has been used to generate cocrystals involving small-molecule templates based on resorcinol that direct intermolecular [2+2] photodimerizations by way of hydrogen bonds. The templates assemble carbon-carbon double $(\mathrm{C}=\mathrm{C})$ bonds of alkenes into the geometry of Schmidt for photoreaction [2]. The vortex grinding 
is used to both initiate the assembly of the templates and olefins to generate the cocrystals, as well as facilitate turnover in photocatalysis.

An important feature of the template method is that the template can be changed, or 'switched', to a derivative in cases where a cycloaddition of a specific olefin does not proceed in up to quantitative yield [3]. The 'template-switching' method relies on having access to resorcinol derivatives that give solids with improved photoreactivity properties. We have also shown that a cocrystal with stacked $\mathrm{C}=\mathrm{C}$ bonds that exhibit dynamic disorder in the form of pedal motion exhibits enhanced reactivity $[4,5]$. The disorder provides a means for reactive $\mathrm{C}=\mathrm{C}$ bonds that crysallize in a 'criss-cross' conformation to rotate to adopt a parallel conformation and react to generate photoproducts in up to quantitative yield. The presence of the dynamic disorder can be considered to effectively circumvent a need to apply the template-switching strategy. The use of resorcinol derivatives as small-molecule catalysts can also be used to enhance [2+2] photodimerizations in solids [1]. Indeed, while many reported template-directed solid-state photodimerizations using resorcinols proceed in near quantitative yield [6], the identification and development of methods that enable olefins to react quantitatively without a need to switch the template still remains a challenge.

With these ideas in mind, we report here an application of dry vortex grinding to improve the yield of a $[2+2]$ photodimerization in the solid state in a case where the yield, although relatively high, was suppressed or 'sluggish' (i.e. 80-90\%). Specifically, we demonstrate that while ultra-violet (UV) irradiation of crystalline (di-Cl-res)·2(1) [4] affords the head-to-head photoproduct 2 in 80-90\% yield (Scheme 1), a post-application of dry vortex grinding [1] to the solid improves the generation of $\mathbf{2}$ to near quantitative yield. We show that the post-application 
of the dry vortex grinding effectively overcomes detrimental effects of static disorder to enable the generation of the cyclobutane photoproduct 2 .
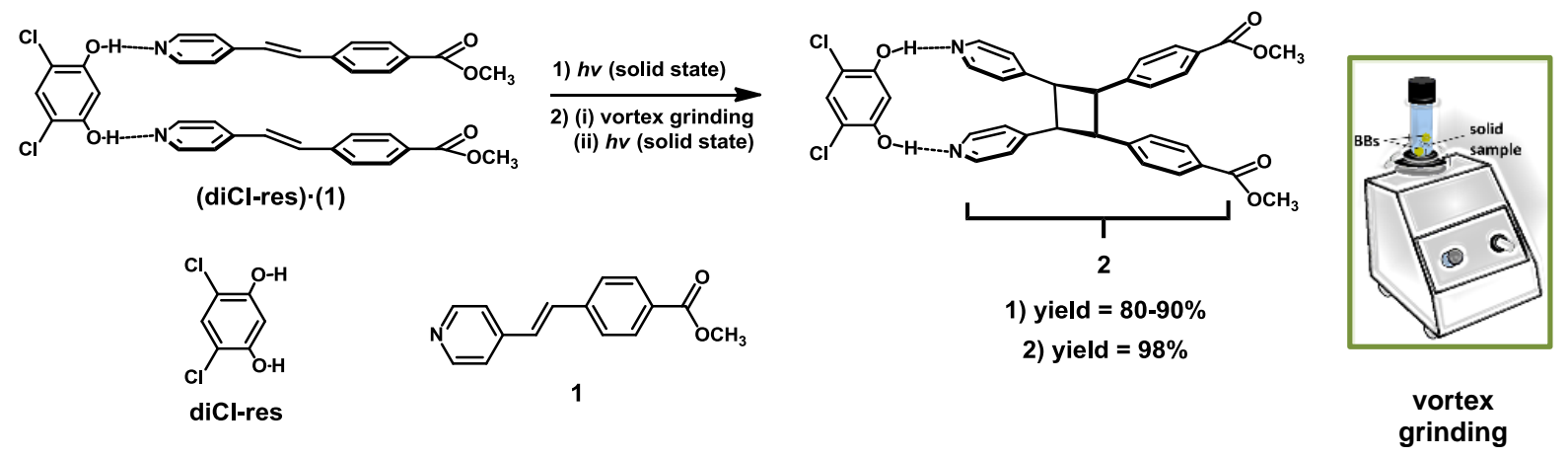

Scheme 1

\section{Experimental}

\subsection{Materials}

All reagents and solvents used were commercially available. Reagent grade ethanol (95\%), 4-pyridinecarboxaldehyde (97\%), methyl (triphenylphosphoranylidene)acetate (98\%), ptoluenesulfonic acid monohydrate (98.5\%), 4,6-dichlororesorcinol (97\%), and dichloromethane ( $\geq 99.8 \%$ ) were purchased from Sigma Aldrich Chemical (St. Louis, MO, USA). All reagents and solvents were used without further purification.

\subsection{Methods}

${ }^{1} \mathrm{H}$ NMR spectra were collected using a Bruker Avance $400 \mathrm{MHz}$ spectrometer using DMSO- $d_{6}$ as a solvent. Photoreactions were conducted using UV-radiation from a $500 \mathrm{~W}$ medium-pressure Hg lamp in an ACE Glass photochemistry cabinet. All solids and the progress of each photoreaction were studied using ${ }^{1} \mathrm{H}$ NMR spectroscopy. Dry vortex grinding was 
conducted using a VWR vortex mixer type Vortex Genie 2. Borosilicate glass vial of dimensions $21 \times 70 \mathrm{~mm}$ were used as sample vials along with premium grade steel BBs $(5 \mathrm{~mm}$ diameter). The melting point of the photoreacted cocrystal (ca $10 \mathrm{mg}$ ) was determined in a capillary tube using a Mel-Temp Melting Point Apparatus 1001D with at a rate of approximately

$5^{\circ} \mathrm{C} / \mathrm{min}$. X-ray powder data were collected at room temperature on a Rigaku Ultima IV X-ray diffractometer between $4^{\circ}$ to $40^{\circ}$ two-theta.

\subsection{Syntheses of $\mathbf{1}$, single crystals of $\mathbf{1}$ and cocrystal (di-Cl-res).2(1)}

The syntheses of $\mathbf{1}$ and cocrystal (di-Cl-res)·2(1) were performed as reported [4]. In a typical experiment, $100 \mathrm{mg}$ of $\mathbf{1}$ was dissolved in $3 \mathrm{ml}$ of ethanol in a sample vial. The resulting solution was then added to a sample vial containing a solution of $50 \mathrm{mg}$ of di-Cl-res in $2 \mathrm{ml}$ of ethanol. Single crystals suitable for X-ray diffraction in the form of blocks formed overnight. Single crystals of $\mathbf{1}$ were grown by slow evaporation from ethanol within a period of two days.

\subsection{Photochemical reactions}

Photoirradiations of (di-Cl-res) 2(1) were carried out using a $500 \mathrm{~W}$ medium-pressure $\mathrm{Hg}$ lamp in an ACE Glass photochemistry cabinet. Thin films of the cocrystals were prepared using mortar-and-pestle grinding. The films were sandwiched between a pair of Pyrex glass plates. Typical sample sizes of (di-Cl-res) $2(\mathbf{1})$ ranged from 50 to $100 \mathrm{mg}$. Samples were irradiated in 8 hour intervals. The dry vortex grinding experiments were conducted using sample sizes on the order of $50 \mathrm{mg}$. Product yields reported according to ${ }^{1} \mathrm{H}$ NMR integrations. 


\subsection{Single-Crystal X-ray Crystallography}

Colorless block-like crystals of $\mathbf{1}$ and (di-Cl-res)·2(1) were individually secured to a cryoloop using Dow Corning grease. Single crystal X-ray diffraction data was collected on a Rigaku SCXMini X-ray diffractometer equipped with a Rigaku Mercury 70 CCD camera. Four independent datasets were collected on the same cocrystal (di-Cl-res).2(1) at 290, 250, 210 and 170 K. Graphite-monochromated Mo-K $\mathrm{K}_{\alpha 1}$ radiation $(\lambda=0.71073 \AA)$ was used in all cases. A data collection strategy to ensure maximum data redundancy was determined using CrystalClear [7]. Data collection, initial indexing, frame integration, Lorentz-polarization corrections, and final cell parameter calculations were carried out using CrystalClear. Multi-scan absorption corrections were performed using REQAB. The structure was solved using direct methods and different Fourier techniques via SHELXT97 [8]. The final structural refinement included anisotropic temperature factors on all non-hydrogen atoms. For all data sets, the positions of the hydrogen atoms were located in the difference map and freely refined. Space groups were unambiguously verified upon analysis of systematic absences using PLATON [9]. In order to achieve the lowered temperatures in the study, the temperature of the crystal was decreased by five degrees every five minutes until the next desired temperature was achieved. After reaching the target temperature, the crystal was allowed to equilibrate for thirty minutes prior to unit cell analysis and commencement of data collection. The percent occupancies of the two components indicative of olefin disorder were quantified using the FVAR command after each was identified in the difference map and refined. Structural parameters for the X-ray data for $\mathbf{1}$ and (di-Clres).2(1) at the different temperatures are included in Table 1. 
Table 1. Crystallographic data for $\mathbf{1}$ and (di-Cl-res) $2(\mathbf{1})$ at different temperatures.

\begin{tabular}{|c|c|c|c|}
\hline compound & 1 & $($ di-Cl-res $) \cdot 2(\mathbf{1})$ & $(\mathrm{di}-\mathrm{Cl}-\mathrm{res}) \cdot 2(\mathbf{1})$ \\
\hline CCDC code & 1430870 & 1010152 & 1010151 \\
\hline formula & $\mathrm{C}_{30} \mathrm{H}_{26} \mathrm{~N}_{2} \mathrm{O}_{4}$ & $\mathrm{C}_{36} \mathrm{H}_{30} \mathrm{Cl}_{2} \mathrm{~N}_{2} \mathrm{O}_{6}$ & $\mathrm{C}_{36} \mathrm{H}_{30} \mathrm{Cl}_{2} \mathrm{~N}_{2} \mathrm{O}_{6}$ \\
\hline formula weight & 478.54 & 657.52 & 657.52 \\
\hline temperature, $\mathrm{K}$ & 293(2) & $290(2)$ & $250(2)$ \\
\hline space group & $\operatorname{Pna2}_{1}$ & $P 2{ }_{1} / n$ & $P 2{ }_{1} / n$ \\
\hline$a, \AA$ & $21.256(3)$ & $15.7045(7)$ & $15.7216(8)$ \\
\hline$b, \AA$ & $15.9858(17)$ & $8.6224(4)$ & $8.6218(4)$ \\
\hline$c, \AA$ & $7.2743(18)$ & $23.5046(16)$ & $23.4946(16)$ \\
\hline$\alpha$, deg & 90.00 & 90.00 & 90.00 \\
\hline$\beta$, deg & 90.00 & $92.650(7)$ & $92.607(7)$ \\
\hline$\gamma, \operatorname{deg}$ & 90.00 & 90.00 & 90.00 \\
\hline volume, $\AA^{3}$ & $2471.8(5)$ & $3179.4(3)$ & $3181.4(3)$ \\
\hline $\mathrm{Z}$ & 4 & 4 & 4 \\
\hline density (calculated), $\mathrm{mg} / \mathrm{m}^{3}$ & 1.286 & 1.374 & 1.373 \\
\hline$\mu, \mathrm{mm}^{-1}$ & 0.086 & 0.255 & 0.254 \\
\hline scan & $\omega$ scan & $\omega$ scan & $\omega$ scan \\
\hline$\theta$ range for data collection, deg & $1.00-25.35$ & $3.10-25.40$ & $3.06-25.35$ \\
\hline reflections measured & 15883 & 27099 & 15485 \\
\hline independent observed reflns. & 4376 & 5811 & 5798 \\
\hline independent reflns. $[I>2 \sigma]$ & 2016 & 4421 & 4467 \\
\hline data/restraints/parameters & $4376 / 21 / 341$ & $5811 / 13 / 537$ & $5798 / 49 / 537$ \\
\hline$R_{\text {int }}$ & 0.0869 & 0.0318 & 0.0262 \\
\hline \multirow[t]{2}{*}{ final $R$ Indices $[I>2 \sigma]$} & $R_{1}=0.0597$ & $R_{1}=0.0378$ & $R_{1}=0.0386$ \\
\hline & $w R 2=0.1397$ & $w R 2=0.0863$ & $w R 2=0.0912$ \\
\hline \multirow[t]{2}{*}{$R$ indices (all data) } & $R_{1}=0.1717$ & $R_{1}=0.0538$ & $R_{1}=0.0544$ \\
\hline & $w R 2=0.2159$ & $w R 2=0.0947$ & $w R 2=0.0977$ \\
\hline goodness-of-fit on $F^{2}$ & 0.978 & 1.035 & 1.018 \\
\hline
\end{tabular}


Table 1. Crystallographic data for $\mathbf{1}$ and (di-Cl-res)·2(1) at different temperatures (cont.).

\begin{tabular}{|c|c|c|}
\hline compound & $($ di-Cl-res $) \cdot 2(\mathbf{1})$ & $($ di-Cl-res $) \cdot 2(\mathbf{1})$ \\
\hline CCDC code & 1010150 & 1010149 \\
\hline $\begin{array}{l}\text { formula } \\
\text { formula weight }\end{array}$ & $\begin{array}{c}\mathrm{C}_{36} \mathrm{H}_{30} \mathrm{Cl}_{2} \mathrm{~N}_{2} \mathrm{O}_{6} \\
657.52\end{array}$ & $\begin{array}{c}\mathrm{C}_{36} \mathrm{H}_{30} \mathrm{Cl}_{2} \mathrm{~N}_{2} \mathrm{O}_{6} \\
657.52\end{array}$ \\
\hline temperature, $\mathrm{K}$ & $210(2)$ & $170(2)$ \\
\hline space group & $P 2_{1} / n$ & $P 2_{1} / n$ \\
\hline$a, \AA$ & $15.6990(8)$ & $15.6872(8)$ \\
\hline$b, \AA$ & $8.6084(4)$ & $8.6088(4)$ \\
\hline$c, \AA$ & $23.4854(16)$ & 23/4798(16) \\
\hline$\alpha, \operatorname{deg}$ & 90.00 & 90.00 \\
\hline$\beta, \operatorname{deg}$ & $92.591(7)$ & $92.572(7)$ \\
\hline$\gamma, \operatorname{deg}$ & 90.00 & 90.00 \\
\hline volume, $\AA^{3}$ & $3170.6(3)$ & $3167.7(3)$ \\
\hline $\mathrm{Z}$ & 4 & 4 \\
\hline density (calculated), $\mathrm{mg} / \mathrm{m}^{3}$ & 1.377 & 1.379 \\
\hline$\mu, \mathrm{mm}^{-1}$ & 0.255 & 0.255 \\
\hline scan & $\omega$ scan & $\omega$ scan \\
\hline$\theta$ range for data collection, deg & $3.06-24.71$ & $3.06-24.11$ \\
\hline reflections measured & 18226 & 15399 \\
\hline independent observed reflns. & 5386 & 5017 \\
\hline independent reflns. $[I>2 \sigma]$ & 4340 & 4153 \\
\hline data/restraints/parameters & $5386 / 49 / 537$ & $5017 / 28 / 537$ \\
\hline$R_{\mathrm{int}}$ & 0.0298 & 0.0241 \\
\hline \multirow[t]{2}{*}{ final $R$ Indices $[I>2 \sigma]$} & $R_{1}=0.0379$ & $R_{1}=0.0371$ \\
\hline & $w R 2=0.0892$ & $w R 2=0.0977$ \\
\hline \multirow[t]{2}{*}{$R$ indices (all data) } & $R_{1}=0.0507$ & $R_{1}=0.0463$ \\
\hline & $w R 2=0.0961$ & $w R 2=0.1033$ \\
\hline goodness-of-fit on $F^{2}$ & 1.053 & 1.073 \\
\hline
\end{tabular}

\section{Results and Discussion}

In a report on an organic protecting group strategy via the organic solid state, we described the ability of $\mathbf{1}$ to assemble with up to 10 different resorcinol derivatives to form 
cocrystals that react to generate $\mathbf{2}$ in up to quantitative yield [4]. The solid (di-Cl-res)·2(1) was a curious case since the cocrystal displayed a relatively short reaction time (i.e. $75 \mathrm{~h}$ ) yet reacted to generate 2 in a high, yet suppressed, $80-90 \%$ yield. At the time of our report, the crystal structure of $\mathbf{1}$ had also not been reported.

\subsection{X-ray Crystal Structures and Solid-State Assemblies}

We have determined that the olefin $\mathbf{1}$, as a pure form, assembles in the solid state to give a photostable solid. As shown in Figure 1, two olefins (molecule A and molecule B) are present in the asymmetric unit, having crystallized in the orthorhombic space group Pna $2_{1}$. The $\mathrm{C}=\mathrm{C}$ of molecule A lies disordered over two sites [site occupancies: 85(1)/15(1)] [5]. Molecule A selfassembles to form a one-dimensional (1D) doubled-stranded polymer along the crystallographic $b$-axis that is sustained by (methyl) $\mathrm{C}-\mathrm{H} \cdots \mathrm{N}($ pyridine) forces $(\mathrm{C} \cdots \mathrm{N}$ separation: $3.27 \AA$ ). Adjacent strands run anti-parallel, with adjacent molecules within the double strand participating in edge-to-face $\pi$-forces. As a result of the assembly process, $\mathrm{C}=\mathrm{C}$ bonds of molecule $\mathrm{A}$ are

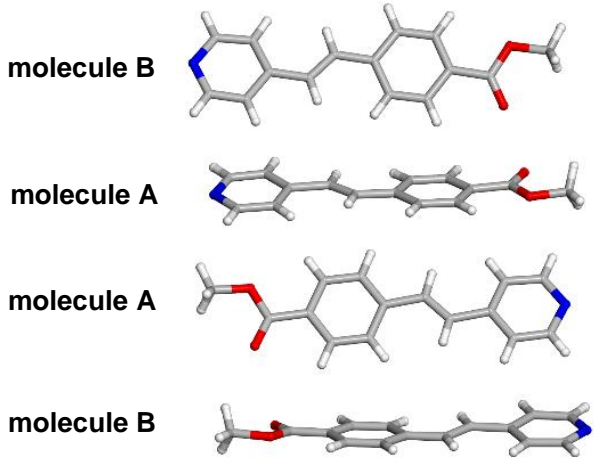

(a)

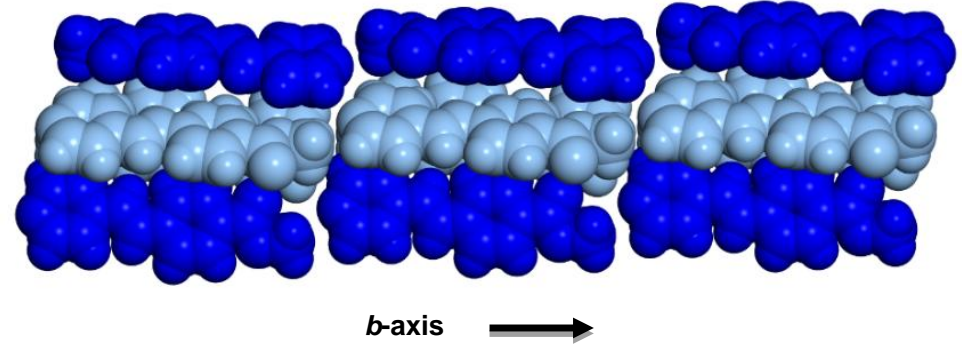

(b)

Figure 1. X-ray crystal structure of 1 showing (a) edge-to-face $\pi$-stacking and (b) $1 \mathrm{D}$ polymer with molecule A (light) and molecule B (dark) (disorder in molecule A omitted for clarity). 
separated by $5.68 \AA$, which lies outside the distance of Schmidt [2] for a photoreaction.

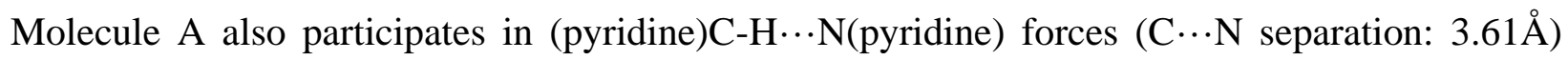
with molecule B, which assembles along the periphery of each double-stranded polymer. The nearest-neighbour $\mathrm{C}=\mathrm{C}$ bonds between molecules $\mathrm{A}$ and $\mathrm{B}$ are separated by $4.67 \AA$. When $\mathbf{1}$ was exposed to UV-radiation for a period of 50 hours, the olefin was determined to be photostable.

Single crystal X-ray diffraction was performed to gain insight into the origin of the suppressed yield of $\mathbf{2}$ in (di-Cl-res).2(1). As shown in Figure 2, the components of (di-Clres) $2(\mathbf{1})$ assemble in the monoclinic space group $\mathrm{P} 2{ }_{1} / \mathrm{n}$ to form a $0 \mathrm{D}$ three-component assembly

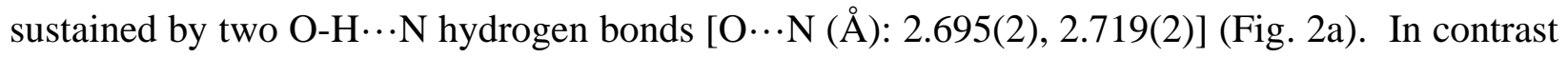
to pure $\mathbf{1}$, the olefins are stacked in a head-to-head geometry such that adjacent $\mathrm{C}=\mathrm{C}$ bonds are separated by $3.86 \AA$. The $\mathrm{C}=\mathrm{C}$ bond of a single stacked molecule of $\mathbf{1}$, similar to pure $\mathbf{1}$, lies disordered over two sites [site occupancies: $0.85(1) / 0.15(1)]$. The $\mathrm{C}=\mathrm{C}$ bond of the majoroccupied site lies parallel to the ordered $\mathrm{C}=\mathrm{C}$ bond within the hydrogen-bonded structure. In the arrangement, the $\mathrm{C}=\mathrm{C}$ bond of the minor-occupied site lies criss-cross with respect to the ordered

(a)

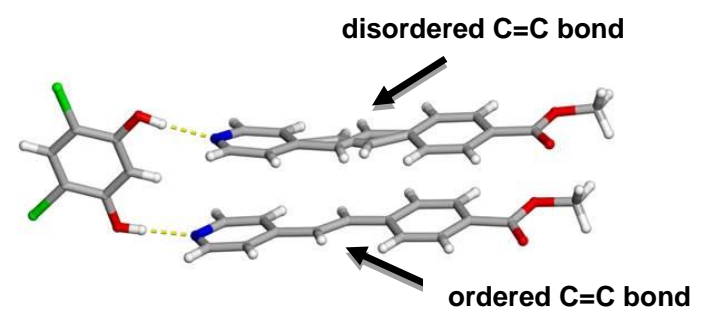

(b)

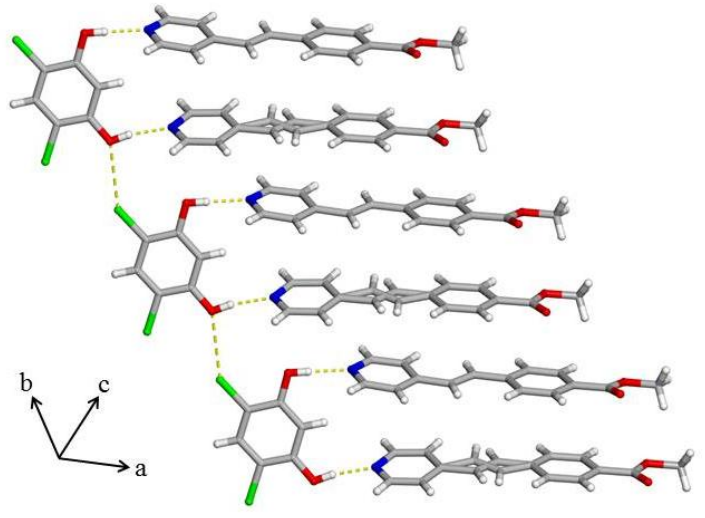

Figure 2. X-ray structure of (di-Cl-res) 2(1): (a) 0D three-component assembly and (b) parallel packing illustrating $\mathrm{O} \cdots \mathrm{Cl}$ forces. 
$\mathrm{C}=\mathrm{C}$ bond. The disorder is, thus, 'unsymmetrical' with respect to the stacking of the two olefins in the $0 \mathrm{D}$ assembly. The three-component assemblies pack to form head-to-head columns, with adjacent assemblies being sustained along the crystallographic $b$-axis by $\mathrm{O} \cdots \mathrm{Cl}$ forces $(\mathrm{O} \cdots \mathrm{Cl}$ separation: $3.17 \AA$ ) [10]. The $\mathrm{C}=\mathrm{C}$ bonds of the nearest-neighbor assemblies are separated by $4.99 \AA$ (Fig. 1b), which is beyond the limit of Schmidt [2] for a photodimerization.

\subsection{Nature of Disorder}

The geometry and site occupancies of the disordered $\mathrm{C}=\mathrm{C}$ bond in the cocrystal (di-Clres) $2(\mathbf{1})$ are consistent with the yield of the [2+2] photodimerization in the solid. In such an environment, the photochemical reaction occurs within the $0 \mathrm{D}$ three-component assemblies to generate 2 in a maximal 85\% yield [4]. Early work of Ohba demonstrated that a criss-cross arrangement of $\mathrm{C}=\mathrm{C}$ bonds can lead to enhanced reactivity involving a pedal-like conformational change to stacked $\mathrm{C}=\mathrm{C}$ bonds [11]. The motion introduces a dynamic element to the topochemical principles of organic solid-state reactions [2,11]. In the case of (di-Cl-res)·2(1), however, the suppressed yield of the photodimerization suggested the disorder of the $\mathrm{C}=\mathrm{C}$ bond of $\mathbf{1}$ in (di-Cl-res).2(1) to be static.

To gain further insight into the nature of the disorder of the $\mathrm{C}=\mathrm{C}$ bond, $\mathrm{X}$-ray diffraction data on a single crystal of (di-Cl-res) -2(1) were collected at different temperatures. Specifically, X-ray data sets were collected at $250 \mathrm{~K}, 210 \mathrm{~K}$, and $170 \mathrm{~K}$. An analysis of the variable temperature X-ray data revealed the site occupancies of the disordered $\mathrm{C}=\mathrm{C}$ bond to remain unchanged (Table 2). The observations are consistent with the $\mathrm{C}=\mathrm{C}$ bond being statically disordered in the solid (Scheme 2). We note there were also minimal changes to the 
intermolecular $\mathrm{C}=\mathrm{C}$ separations, as well as the metrics of the hydrogen- and halogen-bond interactions at the different temperatures.

Table 2. Sites occupancies of the disordered $\mathrm{C}=\mathrm{C}$ bond in (di-Cl-res) $\cdot 2(\mathbf{1})$.

\begin{tabular}{|cc|}
\hline Temperature $(\mathbf{K})$ & Site Occupancy \\
\hline 290 & $0.85(1), 0.15(1)$ \\
250 & $0.85(1), 0.15(1)$ \\
210 & $0.86(1), 0.14(1)$ \\
170 & $0.86(1), 0.14(1)$ \\
\hline
\end{tabular}

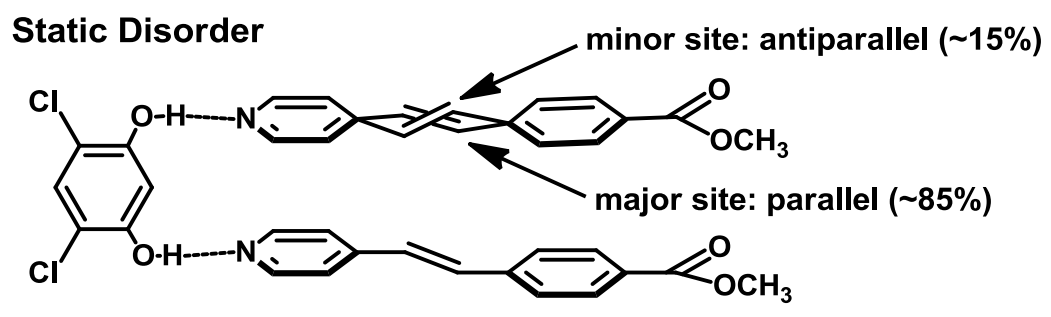

Scheme 2

\subsection{Dry Vortex Grinding}

While 2 forms from (di-Cl-res).2(1) in $80-90 \%$ yield, we have determined that a postapplication of dry vortex grinding [1] to the reacted solid improves the yield of 2 to near quantitative yield $[12,13]$. That mechanochemical grinding enhance reactivity of criss-crossed olefins in a crystalline hydrogen-bonded metal complex has been reported by Vittal [12]. Thus, a powdered crystalline sample of (di-Cl-res) $2(\mathbf{1})$ was placed between two plates and exposed to UV radiation (broadband $\mathrm{Hg}$ lamp) for a period of 50 hours (yield: 85\%). The sample was then subjected to dry vortex grinding for a period of approximately 5 minutes. The sample was then exposed to UV radiation for an additional 50 hours. A ${ }^{1} \mathrm{H}$ NMR spectrum of the ground solid 
exposed to the UV-radiation revealed the yield of $\mathbf{2}$ to increase to near quantitative yield (98\%) [14].

To understand how the vortex grinding supports the improved yield of $\mathbf{2}$ in photoreacted (di-Cl-res) 2(1), the solid was studied using powder X-ray diffraction. As shown in Figure 3, powder diffractograms were collected before and after the application of the vortex grinding on photoreacted (di-Cl-res)·2(1). For comparisons, a calculated diffractogram of the X-ray structure

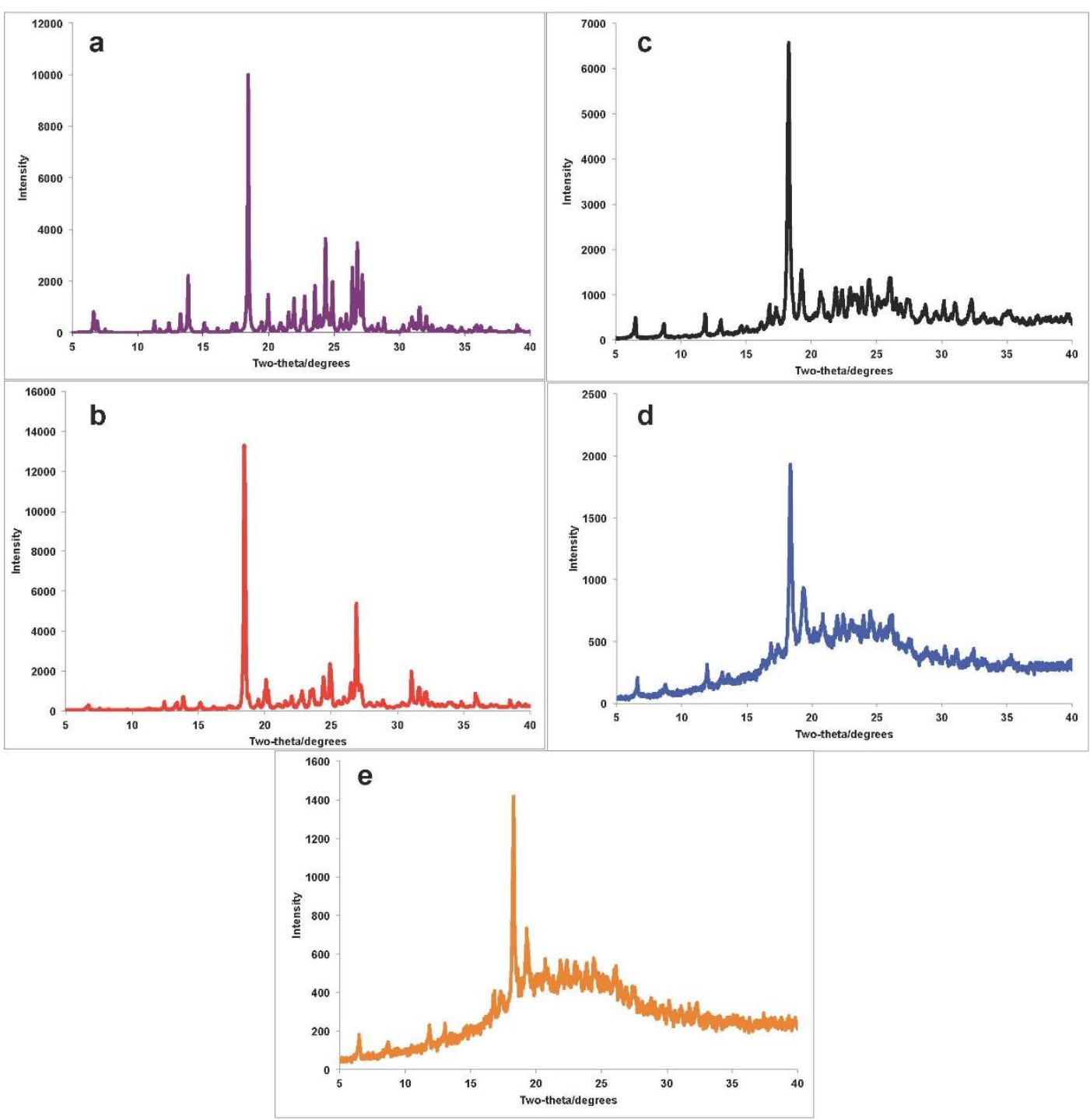


Figure 3. X-ray diffraction data for (di-Cl-res)·2(1): (a) calculated structure, (b) powder prior to photoreaction, (c) powder prior to grinding (85\% yield), (d) powder after grinding, and (e) powder at end of photochemical reaction (98\% yield).

of (di-Cl-res) -2(1) (Fig. 3a), as well as diffractograms at the beginning and end of the irradiation experiments (Figs. 3b,e), are given. From the PXRD data, it was determined that crystalline (diCl-res).2(1) undergoes a change in phase following the initial application of the UV-radiation (85\% yield) (Fig. 3c). The observation is consistent with many reported solid-state photodimerizations [15], with changes in phase being attributed to losses of accumulated stress and strain in the solids. The PXRD data also showed that there was no apparent change in phase following the application of the vortex grinding (Fig. 3d). The structure of the solid also appeared to be retained following the second application of the UV-radiation (98\% yield) (Fig. 3e). Given that a change in phase was not observed following the vortex grinding, we believe that the enhanced reactivity of $\mathbf{1}$ can be explained according to the disordered $\mathrm{C}=\mathrm{C}$ bond in (diCl-res)·2(1) undergoing a mechanically-induced change in conformation to a reactive orientation in the solid (Scheme 2) [1c,12]. Such a change in conformation [5] may occur within the OD hydrogen-bonded assembly [1c] and may be assisted by a recrystallization of the reactive complex and/or photoproduct [1c]. We note that, in a separate experiment, simple heating of the partially reacted solid ( $85 \%$ yield) to $80{ }^{\circ} \mathrm{C}$ (see, supplementary material) and a subsequent application of UV-radiation did not result in the improved yield. The observation supports that mechanical energy alone is responsible to achieve the enhanced reactivity of $\mathbf{2}$ in (di-Clres) $2(\mathbf{1})$. 


\section{Conclusions}

In this report, we have shown disorder of an olefin within a OD hydrogen-bonded assembly to contribute to a suppressed yield of a solid-state [2+2] photodimerization. Variable temperature X-ray structure data has revealed the disorder to be static. We have also demonstrated that dry vortex grinding can be used to improve the yield of the photoreaction. We are currently studying the scope of the vortex approach as a means to improve yields of photodimerizations in solids that are mediated using resorcinol templates.

\section{Acknowledgements}

We are grateful to Webster University for a Faculty Research Grant and Faculty Development Fund (R.H.G) as well as the National Science Foundation (L.R.M., DMR1408834) for funding. The authors would also like to acknowledge California State University San Marcos for the funds to purchase the Rigaku SCXMini X-ray diffractometer.

\section{References}

1. (a) J. Stojakovic, B.S. Farris, L.R. MacGillivray, Liquid-assisted vortex grinding supports the single-step solid-state construction of a [2.2]paracyclophane. Faraday Discuss. 170 (2014) 35; (b) J. Stojakovic, B.S. Farris, L.R. MacGillivray, Vortex grinding for mechanochemistry: application for automated supramolecular catalysis and preparation of a metal-organic framework. Chem. Commun. 48 (2012) 7958; (c) A.N. Sokolov, D.-K. Bučar, J. Baltrusaitis, S.X. Gu, L.R. MacGillivray, Supramolecular catalysis in the organic solid state via dry grinding. Angew. Chem., Int. Ed. 49 (2010) 4273.

2. G.M.J. Schmidt, Photodimerization in the solid state. Pure Appl. Chem. 27 (1971) 647. 
3. T. Friščić, L.R. MacGillivray, Template-switching: A supramolecular strategy for the quantitative, gram-scale construction of a molecular target in the solid state. Chem. Commun. (2003) 1306.

4. E. Elacqua, P. Kaushik, R.H. Groeneman, D.-K Bučar, J.C. Sumrak, L.R. MacGillivray, A supramolecular protecting group strategy applied in the organic solid state. Angew. Chem., Int. Ed. 51 (2012) 1037.

5. J. Harada, K. Ogawa, Pedal motion in crystals. Chem. Soc. Rev. 38 (2009) 2244.

6. L.R. MacGillivray, G.S. Papaefstathiou, T. Friščić, T.D. Hamilton, D.-K. Bučar, Q. Chu, D.B. Varshney, I.G. Georgiev, Supramolecular control of reactivity in the solid state: From templates to ladderanes to metal-organic frameworks. Acc. Chem. Res. 41 (2008) 280.

7. J.W. Pflugrath, The finer things in X-ray diffraction data collection. Acta Crystallogr. D55 (1999) 1718.

8. G.M. Sheldrick, A short history of SHELX. Acta Crystallogr. A64 (2008) 112.

9. A.L. Speck, PLATON, A Multipurpose Crystallographic Tool, Utrecht University, The Netherlands, 2001.

10. A. Mukherjee, S. Tothadi, G. R. Desiraju, Halogen bonds in crystal engineering: like hydrogen bonds yet different. Acc. Chem. Res. 47 (2014) 2514.

11. S. Ohba, H. Hosomi, Y. Ito, In situ x-ray observation of pedal-like conformational change and dimerization of trans-cinnamamide in cocrystals with phthalic acid. J. Am. Chem. Soc. 123 (2001) 6349.

12. A.M.P. Peedikakkal, J.J. Vittal, Solid-state photochemical [2+2] cycloaddition in a hydrogen-bonded metal complex containing several parallel and crisscross $\mathrm{C}=\mathrm{C}$ bonds. Chem. Eur. J. 14 (2008) 5329. 
13. For a report on grinding to achieve a [2+2] photodimerization in the solid state involving a cucurbit[8]uril, see: B.C. Pemberton, A. Ugrinov, J. Sivaguru, Evaluating photodimerization of 6-methylcoumarin mediated by cucurbit[8]uril through mechanical grinding - Supramolecular effects of additives. J. Photochem. Photobiol., A: Chem 255 (2013) 10.

14. The pyridyl and cyclobutane protons exhibit slight line broadening and shifts consistent with the presence of hydrogen bond and/or acid-base exchange [4].

15. (a) K. Biradha, R. Santra, Crystal engineering topochemical solid state reactions. Chem. Soc. Rev. 42 (2013) 950; (b) V. Ramamurthy, K. Venkatesan, Photochemical reactions of organic crystals. Chem. Rev. 87 (1987) 433. 